The First Three Minutes. By Steven Weinberg. Pp. 188. (Basic Books: New York, March 1977; Andre Deutsch: London, September 1977.) \$8.95; $£ 4.50$.

DURING the twentieth century, cosmology has been transformed from metaphysics to physics, creating one of the most exciting arenas for scientific investigation. The fundamental laws of physics on the one hand and dramatic progress in observational astronomy on the other make it possible to reconstruct a general evolutionary picture of the Universe's past history with some certainty.

If one takes the present structure and reverses the sense of the observed expansion then extrapolation backwards in time reveals increasingly denser and hotter cosmic epochs. The times of important event strata can be established in the manner of geology until eventually a 'big bang' is envisaged $10,000 \mathrm{Myr}$ ago. This hot scenario for cosmogeny is based on the existence of a cosmic background radiation field, predicted by Gamow in 1948, and detected at microwavelengths by Penzias and Wilson in 1964. The photons of this radiation background are now unanimously interpreted as a vestigial remnant of the extreme conditions prevailing in the primordial fireball itself, when matter was completely ionised and the Universe a high energy physicist's paradise.

The increasing temperature as we move further into the past towards the 'bang' ensures that cosmology can provide a remarkable theoretical laboratory for particle physics. Of particular importance has been the realisation that during the first few minutes of expansion from the 'bang', physical conditions were expedient for the fusion of light nuclei having distinctive abundances. The fossilised abundances of helium and deuterium have been detected in precisely the range predicted by the simplest models and reassure us that a view into the early opaque phase of the Universe's life is possible by indirect means. The contingency of thermodynamic equilibrium in the first few seconds washes away all the uncertainties surrounding the initial state and enables laboratory physics to develop a fairly complete description of events.
Steven Weinberg has again very successfully turned his attention from the world of microphysics, where his contributions are well known, to cosmology. The First Three Minutes is a clear and challenging description of the Universe's early evolution suitable for the informed layman and practising scientist alike. The author has vividly explained the essential concepts and physical processes involved, without recourse to mathematics or the details of general relativity, by introducing the necessary physical ideas as his story unfolds. One of the remarkable features of the book is the manner in which the author has maintained the rigour of his physical argument throughout, never sacrificing logical continuity in the interests of speed or oversimplification.

The first three chapters are partially historical in content, describing the discoveries of the universal expansion and microwave background radiation. Unfortunately, no explanation of the crushing blow dealt to the steady-state theory by the latter observations is provided. Such a description is essential in a work intended for the nonspecialist, who often believes this theory still to be in strong competition with the 'big bang' cosmologies.

In the fourth chapter the physics of black-body radiation is described with great clarity, together with the conserved quantities necessary to specify the microstructure of the Universe uniquely. This careful build-up prepares the reader for the key chapter in which the phenomena peculiar to the first three minutes of expansion from the origin are expounded frame by frame. The final chapter is an ex- cursion into the history of science in an effort to discover the reasons for scientists' procrastination in establishing this picture when the necessary observational and theoretical ingredients were apparently available.

Criticisms of the book are minor. Perhaps a little more space could have been allocated to the first three minutes rather than the past fifty years. The opportunity could then have been taken to mention Hawking's exciting work on primordial black holes or problems surrounding the existence of a singularity in spacetime. Some more clear diagrams displaying the evolutionary phases considered and their generic phenomena would also have been welcome landmarks to establish the overall panorama more vividly. Finally, a little more space might have been devoted to the conceptual problems associated with the standard model; these latter are of great intrinsic interest and motivate much current work in cosmology. Weinberg more than atones, however, for these failings with many fascinating digressions into points of basic physics and by a lucidity that makes for compelling reading. The provision of a glossary of technical terms, detailed index and bibliography are further attractive features of what will surely become a landmark in the exposition of the subject.

This book can be strongly recommended to all as the place to begin or reinforce their education in 'prehistoric' cosmology.

John Barrow is a Junior Research Lecturer at Christ Church College, and a member of the Department of Astrophysics at the University of Oxford, UK.

\title{
Nerve, synapse and muscle
}

Electrobiology of Nerve, Synapse and Muscle. Edited by J. P. Reuben, D. P. Purpura, M. V. L. Bennett and E. R. Kandel. (Raven: New York, 1976.) \$30.

WrITTEN by his students and associates, this Festschrift volume in honour of Harry Grundfest contains a collection of recent studies on nerve, synapse and muscle. As might be expected from Grundfest's own interests, this volume ranges widely and provides fascinating glimpses of the ways in which an im- portant area of biology has grown and developed. The chapters are uniformly interesting and no electrobiologist could pick up this book and fail to learn something new. Cell physiologists around the world will be delighted that Harry Grundfest's contribution has been honoured in this way.

P. F. Baker

P. F. Baker is Professor of Physiology at King's College, University of London, UK. 